\title{
Lipoprotein(a) and the risk of atrial fibrillation - is there a link
}

\author{
Marcin A. Bartlomiejczyk ${ }^{\mathrm{A}}$, Peter Penson ${ }^{\mathrm{B}}$, Maciej Banach ${ }^{\mathrm{A}}$
}

A - Department of Hypertension, WAM, University Hospital in Lodz, Medical University of Lodz, Zeromskiego 113, 90-549 Lodz, Poland; \#maciej.banach@icloud.com

B - School of Pharmacy \& Biomolecular Sciences, Liverpool John Moores University, Byrom Street, Liverpool, L3 3AF, UK

Lipoprotein(a) $[\mathrm{Lp}(\mathrm{a})]$ is a lipoprotein moiety with similar lipid composition to low-density lipoprotein (LDL). It is composed of apolipoprotein B100 (ApoB100) and apolipoprotein(a) (apo(a)) bound by a single disulphide bridge ${ }^{[1,2]}$. Apo(a) is a large hydrophilic glycoprotein, which shares $80-90 \%$ homology with plasminogen. Homology was confirmed by sequencing of cloned human apo(a) $\mathrm{CDNA}^{[3]}$. The role of $\mathrm{Lp}(\mathrm{a})$ in health and disease is not yet fully understood. It has been suggested that $\mathrm{Lp}(\mathrm{a})$ has prothrombotic properties. $\mathrm{Lp}$ (a) competes with plasminogen for binding to endothelial and mononuclear cells as well as to platelets, thereby reducing the conversion of plasminogen to plasmin and inhibiting fibrinolysis ${ }^{[4]}$. Furthermore, elevated $L p(a)$ has been associated with increased risk for the development of cardiovascular $(\mathrm{CV})$ and cerebrovascular atherosclerotic disease ${ }^{[5,6]}$. Interestingly, raised levels of $L p(a)$ have been observed in women with polycystic ovary syndrome (PCOS) and patients suffering from venous thromboembolism $^{[5]}$. In 1998, Igarashi et al. suggested that serum $\mathrm{Lp}$ (a) concentration was a significant independent positive predictor for left atrial thrombus in patients with chronic atrial fibrillation $(\mathrm{AF})^{[3]}$.

$\mathrm{AF}$ is a major cause of morbidity and hospitalization. Moreover, AF is an important risk factor for thromboembolic complications and cerebrovascular diseas $\mathrm{e}^{[7]}$. Some available data have demonstrated that AF is associated with ischemic heart disease (IHD), arterial hypertension, heart failure (HF), obesity and metabolic syndrome (MetS) ${ }^{[8]}$. In recent years, $\mathrm{Lp}$ (a) has emerged as an important CV risk factor ${ }^{[4]}$. In several studies and meta-analysis, Lp(a) has been associated with an increased incidence of coronary heart disease (CHD). Igarashi et al. found that patients with $\mathrm{AF}$ and left atrial thrombus had elevated levels of $\mathrm{Lp}(\mathrm{a})^{[3]}$. In vitro and in vivo studies support the hypothesis that $L p(a)$ has thrombogenic effects and may promote thrombus formation in the left atrium in patients with chronic AF, by inhibiting the fibrinolytic system ${ }^{[3]}$. However, Diaz-Peromingo et al. in 2006 found no statistically significant differences between the groups of patients with or without $\mathrm{AF}$, suggesting that $\mathrm{Lp}(\mathrm{a})$ levels are not clearly associated with $\mathrm{AF}^{[5]}$. This might be partly explained by the hypothesis that Apo(a) (which is similar in structure to plasminogen) interferes with fibrinolytic system ${ }^{[8]}$. Aronis et al ${ }^{[9]}$ found no association between circulating levels of $\mathrm{Lp}(\mathrm{a})$ at baseline and AF incidence. These results were similar among whites and blacks and men and women ${ }^{[9]}$. In 2011 Naji and Sabovic also did not find any positive correlation between $\mathrm{Lp}(\mathrm{a})$ and $\mathrm{AF}^{[8]}$. Their results were similar to data obtained by Diaz-Peromingo et al., who did not find any relationship between Lp(a) levels and $\mathrm{AF}^{[5]}$. Naji and Sabovic compared results between a group of patients with $\mathrm{AF}$ and a control group with sinus rhythm. Each group was comprised of 101 patients. In the AF group, they found a higher total cholesterol/high density lipoprotein (HDL) ratio, but there were no different between the groups in terms of $L p(a)$ levels. Their results were probably influenced by ethnic characteristics ${ }^{[5]}$. Aronis et al. tried to minimalize the risk of false negative/positive results caused by ethnic characteristics by recruiting 9,908 participants (43.9\% men, $21.6 \%$ black) and their study design enabled investigation of statistical interaction by race and sex. Furthermore, Lp(a) levels were measured with a well-validated assay that is robust to different Apo(a) isoform sizes ${ }^{[9]}$. On the other hand, Wen-Jia et al. [2016], found a significant association between Lp(a) levels and AF. These authors demonstrated association of elevated Lp(a) levels with increasing risk of new-onset AF currency $(p<0.05,95 \%$, CI (1.005-7.22)). They followed 534 patients with chronic heart failure without AF from their admission until a new occurrence of $\mathrm{AF}^{[10]}$. The relationship between $\mathrm{Lp}(\mathrm{a})$ and AF might arise from a connection between $\mathrm{Lp}(\mathrm{a})$ and inflammation and the fact that its similarity with plasminogen results in interference with the fibrinolytic system. Moreover, differences between the results reported by Wen-Jia and those reported by Naji and by Sabovic might be due to different baseline characteristics of the enrolled patients such as cardiac function, lipid levels and prevalence of coronary heart disease ${ }^{[8,10]}$. Additionally, the study conducted by Wen-Jia et al. ${ }^{[2]}$, was carried on the Chinese population, Aronis et al..$^{[9]}$, included white and black participants, whereas the Diaz-Peromingo study population was comprised of white European patients ${ }^{[5]}$. Ethnic differences in $\mathrm{CV}$ risk factors have been previously described. Higher levels of Lp(a) have been reported in Hispanics and African Americans than in white 
Americans, Europeans or Asians. Blacks have approximately 3-fold higher Lp(a) levels compared with whites, and women have higher Lp(a) levels by 15\% - 20\% compared with men. Despite higher Lp(a) levels, Blacks and women have a lower incidence of $\mathrm{AF}$, and little is known about whether race or sex modify $\mathrm{Lp}(\mathrm{a})$-related risk ${ }^{[10,11]}$. It has been suggested that genetic factors account for much of the variation in serum $\mathrm{Lp}(\mathrm{a})$ of different populations. The prevalence of AF also varies greatly between ethnic groups. Whites have a greater frequently of AF than Black African, Black Caribbean or Asian populations ${ }^{[12,13]}$. Therefore, population studies which have included white European patients may report higher prevalence of AF and lower levels of L $\mathrm{p}(\mathrm{a})$. These special characteristics of populations may in part describe the lack of association between variables in several studies. Therefore further studies are still needed to elucidate the role of $\mathrm{Lp}(\mathrm{a})$ in $\mathrm{AF}$ in different racial groups.

\section{References}

1. Ferretti G, Bacchetti T, Johnston TP, Banach M, Pirro M, Sahebkar A. Lipoprotein(a): A missing culprit in the management of athero-thrombosis? J Cell Physiol. 2018 Apr;233(4):2966-2981

2. Wen-Jia L., Ming-Hong L., Rui Y., Yu-Qi C., Lei Y., Shi-Liang J., Lian-Qun C., Li-Ming C. The prospective effect of lipoprotein(a) on new-onset atrial fibrillation in patients with chronic heart failure. Int J Clin Exp Med. 2016, 9(9), 18316-18323.

3. Igarashi Y., Yamaura M., Ito M., Inuzuka H., Ojima K., Aizawa Y. Elevated serum lipoprotein(a) is a risk factor for left atrial thrombus in patients with chronic atrial fibrillation: A transesophageal echocardiographic study. Am Heart J. 1998, 136, 965-971.

4. Kotani K., Serban M.C., Penson P., Lippi G., Banach M. Evidence-based assessment of lipoprotein(a) as a risk biomarker for cardiovascular diseases - Some answers and still many questions. Crit Rev Clin Lab Sci. 2016, 53(6): 370-8.

5. Díaz-Peromingo J.A., Albán-Salgado A., García-Suárez F., Sánchez-Leira J., Saborido-Froján J., Iglesias-Gallego M. Lipoprotein(a) and lipid profile in patients with atrial fibrillation. Med Sci Monit. 2006, 12(3), 122-125.

6. 6. Banach M. Lipoprotein (a)-We Know So Much Yet Still Have Much to Learn .... J Am Heart Assoc. 2016;5(4):e003597.

7. Shariff N, Desai RV, Patel K, et al. Rate-control versus rhythm-control strategies and outcomes in septuagenarians with atrial fibrillation. Am J Med. 2013 Oct;126(10):887-93.

8. Naji F., Sabovic M. Lipoprotein(a) and inflammation in patients with atrial fibrillation after electrical cardioversion. J Negat Results Biomed. 2011;10:15.

9. Aronis K, Zhao D, Hoogeveen RC, et al. Associations of Lipoprotein(a) Levels With Incident Atrial Fibrillation and Ischemic Stroke: The ARIC (Atherosclerosis Risk in Communities) Study. J Am Heart Assoc. 2017, 6(12), 1-12.
10. Lee T.C., O'Malley P.G., Feuerstein I., Taylor A.J. The prevalence and severity of coronary artery calcifi cation on coronary artery computed tomography in black and white subjects. J AM Coll Cardiol, 2003, 41(1), 39-44.

11. Matthews K.A., Sowers M.F., Derby C.A., Stein E., Miracle-McMahill H., Crawford S.L., Pasternak R.C. Ethnic differences in cardiovascular risk factor burden among middle-aged women: Study of Women's Health Across the Nation (SWAN). Am Heart J, 2005, 149(6), 1066-73.

12. Hajat C., Tilling K., Stewart J.A., Lemic-Stojcevic N., Wolfe C.D. Ethnic differences in risk factors for ischemic stroke: a European case-control study. Stroke, 2004, 35(7): 1562-67.

13. Sacco R.L., Boden-Albala B., Abel G., Lin I.F., Elkind M., Hauser W.A., Paik M.C., Shea S. Race-ethnic disparities in the impact of stroke risk factors: the northern Manhattan stroke study. Stroke, 2001, 32(8): 1725-31. 